\title{
Strain effects on optical properties of pyramidal InAs/GaAs quantum dots
}

\author{
M.K. Kuo*, T.R. Lin, B.T. Liao, C.H. Yu ${ }^{1}$ \\ Institute of Applied Mechanics, National Taiwan University, Taipei, 106 Taiwan, ROC
}

Available online 23 November 2004

\begin{abstract}
Strain distribution and optical properties in a self-assembled pyramidal InAs/GaAs quantum dot grown by epitaxy are investigated. A model, based on the theory of linear elasticity, is developed to analyze three-dimensional induced strain field. In the model, the capping material in the heterostructure is omitted during the strain analysis to take into account the sequence of the fabrication process. The mismatch of lattice constants is the driving source of the induced strain and is treated as initial strain in the analysis. Once the strain analysis is completed, the capping material is added back to the heterostructure for electronic band calculation. The strain-induced potential is incorporated into the threedimensional steady-state Schrödinger equation with the aid of Pikus-Bir Hamiltonian with modified Luttinger-Kohn formalism for the electronic band structure calculation. The strain field, the energy levels and wave functions are found numerically by using of a finite element package FEMLAB. The energy levels as well as the wave functions of both conduction and valence bands of quantum dot are calculated. Finally, the transition energy of ground state is also computed. Numerical results reveal that not only the strain field but also all other optical properties from current model show significant difference from the counterparts of the conventional model.
\end{abstract}

(C) 2004 Elsevier B.V. All rights reserved.

PACS: 73.61.-r; 73.21.La; 73.40.Gk

Keywords: Quantum dots; InAs/GaAs; Lattice constants mismatch; Induced strain; Pikus-Bir Hamiltonian

\section{Introduction}

Quantum dots (QDs), owing to its own threedimensional quantum confinement, are found to

\footnotetext{
*Corresponding author. Fax: 2-3366-5631.

E-mail address: mkkuo@ntu.edu.tw (M.K. Kuo).

${ }^{1}$ Now at Chung-San Institute of Science and Technology, Taoyuan 325, Taiwan, ROC.
}

have delta-function distributions of density of states, discrete energy levels, "atom-like" electronic states, etc., and hence have attracted substantial attention recently [1]. Self-assembled QDs (SAQDs) formed by strained epitaxy have shown the promising result in having a large array of quantum dots. On the other hand, SAQD formation is commonly observed in large mismatch epitaxy of chemically similar materials $[1,2]$. 
Strain fields inside quantum-dot hetrostructures strongly affect the electronic properties and hence the optoelectronic properties in the vicinity of dots [3]. To understand the strain effects on optical properties of quantum dots, determination of the induced strain field in the dots and the surrounding matrix is necessary. Finite element analysis has been used to investigate the strain field in the quantum dot [4-6].

A single square-based pyramidal self-assembled InAs quantum dot buried in GaAs matrix is considered in the article as depicted in Fig. 1. The InAs is grown a thin wetting layer on $\left(\begin{array}{lll}0 & 0 & 1\end{array}\right) \mathrm{GaAs}$ substrate during heteroepitaxy, followed by coherent island formation. Finally, the quantum-dot island is subsequently covered by additional deposition of substrate materials.

\section{Strain field}

Epitaxially grown SAQD heterostructures often consist of several materials with different lattice constants. It gives rise to strain fields in a quantum-dot nanostructure, and affects the optical properties of the quantum dots [6]. In-plane lattice, mismatch parameter is usually defined as

$\left(\varepsilon_{0}\right)_{x x}=\left(\varepsilon_{0}\right)_{y y}=\frac{a_{\mathrm{s}}-a_{\mathrm{d}}}{a_{\mathrm{d}}}$,

where $a_{\mathrm{s}}$ and $a_{\mathrm{d}}$ are the lattice constants of the substrate and the dot materials, respectively, i.e.,

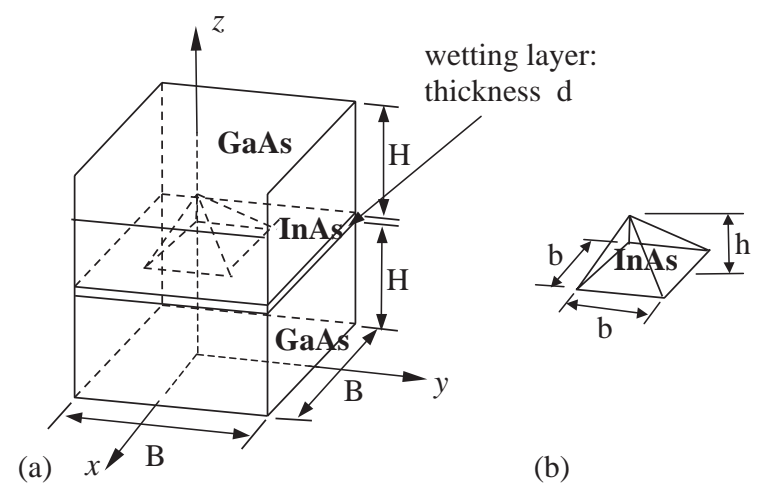

Fig. 1. Geometry of the quantum-dot system. (a) InAs/GaAs QD heterostructure, and (b) InAs quantum-dot island, where $B=H=30 \mathrm{~nm}, d=0.5 \mathrm{~nm}, b=12 \mathrm{~nm}$, and $h=6 \mathrm{~nm}$.
GaAs and InAs, and they will be taken as 0.565 and $0.605 \mathrm{~nm}$, respectively, in the numerical computation of this article.

Notice that, the mismatch of lattice constants will induce further elastic deformation in the whole nanostructure system, namely, in both the substrate and the quantum-dot island. Thus the lattice mismatch parameter defined in Eq. (1) is not the only strain in the quantum-dot island. It will instead be treated as in-plane initial strain in the latter finite element analysis.

The out-of-plane initial strain, on the other hand, has never yet been as conclusive as its inplane counterparts. It is a common belief that the in-plane initial strain in the epitaxy process will certainly accomplish with out-of-plane strain. In the article, the out-of-plane initial strain is taken as if it were strained elastically and in plane-stress state, which leads to

$\left(\varepsilon_{0}\right)_{z z}=-\frac{2 C_{12}}{C_{11}}\left(\varepsilon_{0}\right)_{x x}$.

Here both GaAs and InAs are treated as cubic materials with three independent elastic moduli each, namely, $C_{11}, C_{12}$, and $C_{44}$.

In the article, parameters $\varepsilon_{0}$ 's are regarded as the initial normal strains in the $x, y$, and $z$ directions in the latter finite element calculation. These initial strains in the wetting layer and the quantum-dot island will further induce the strain field in a SAQD system.

According to the theory of linear elasticity, the relationship among stresses $\sigma_{i j}$, total strains $\varepsilon_{k l}$, and initial strains can be expressed as

$\sigma_{i j}=C_{i j k l}\left[\varepsilon_{k l}-\left(\varepsilon_{0}\right)_{k l}\right], \quad i, j, k, l=x, y, z$,

where $C_{i j k l}$ is the elastic moduli, and $\varepsilon_{0}$ is the normal initial strain described in Eqs. (1) and (2).

The wetting layer grows on the substrate during the quantum-dot fabrication process, and then further embedded in the capping material after quantum-dot island formation, as shown in Fig. 1. Since the capping material is deposited after the quantum-dot island has been formed, it should have never experience the deformation of quantum dot in the self-assembled process. Hence, the capping material is not included in the strain analysis. 
In the stage of strain analysis, the quantum-dot heterostructure system is considered to consist of only quantum dot, wetting layer and substrate but not capping material. The mismatch of lattice constants of the heterostructure is the driving source for the induced strain. Once the strain analysis has been completed, the capping layer is then added into the system as if it is deposited after quantum-dot formation.

The adding of capping material is only for the purpose of solving the Schrödinger equation to obtain the electronic structure of the nanostructure system. This proposed model partially takes the sequence of fabrication process into account as compared to the conventional model where the capping material is considered as a body with other materials.

Figs. 2 and 3 show the normal strain $\varepsilon_{x x}$ and $\varepsilon_{z z}$, respectively, along the $z$-axis in quantum-dot nanostructures. It is not surprising that these strain components are discontinuous at the interface of the wetting layer and the substrate (i.e. InAs/GaAs interface).

Notice that, as seen from the figures, the strain filed $\varepsilon_{x x}$ inside the dot is compressive while $\varepsilon_{x x}$ is tensile. These reflect the fact that the lattice constant of InAs is larger than that of GaAs. From Figs. 2-3, it is easy to conclude that strain distributions obtained from the conventional and proposed models lead to significant difference.

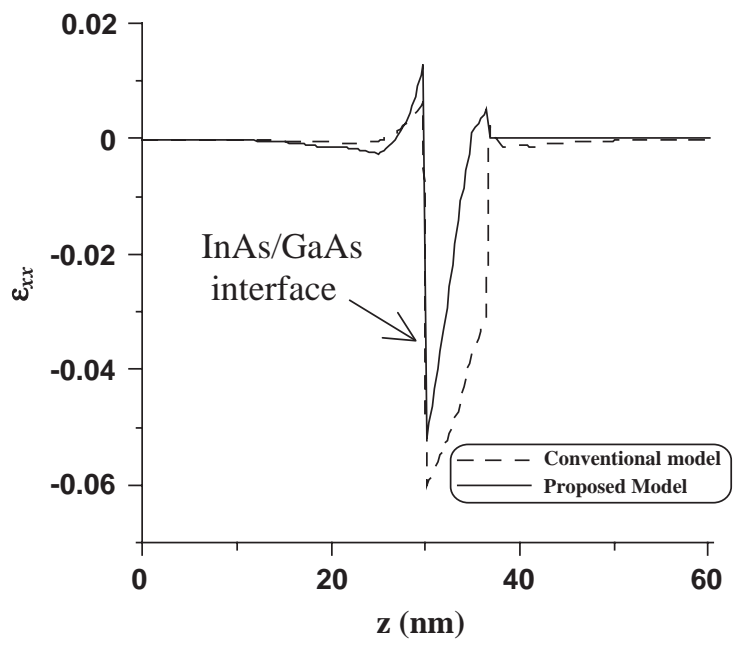

Fig. 2. Strain component $\varepsilon_{x x}$ along the $z$-axis.

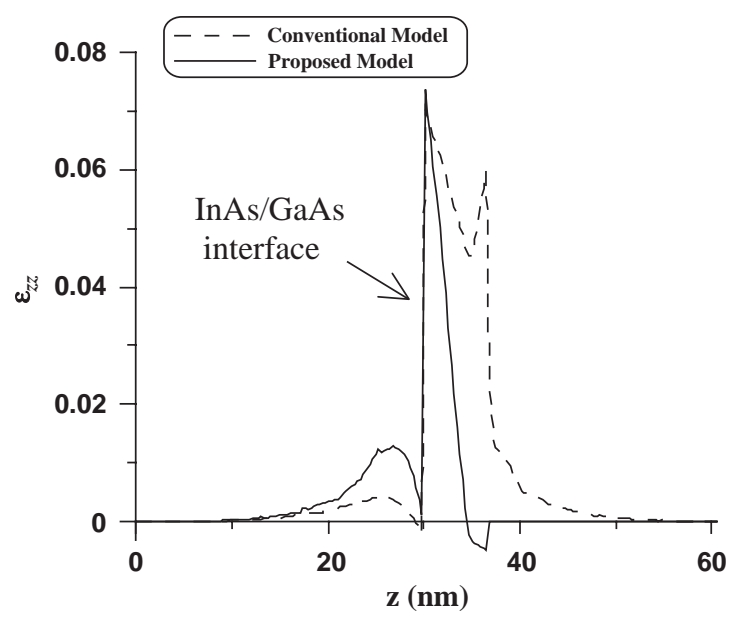

Fig. 3. Strain component $\varepsilon_{z z}$ along the $z$-axis.

\section{Optical properties}

For strained quantum-dot nanostructures, the confinement potential can be written as a sum of energy offsets of the conduction band (or valence band), $V_{\text {band, }}$ and the strain-induced potential, $V_{\text {strain }}$, as

$V(\vec{r})=V_{\text {band }}(\vec{r})+V_{\text {strain }}(\vec{r})$.

Since strain effects induce an extra potential field, it then alters the band structure and optical properties of the quantum-dot system. In spite of inhomogeneous distribution of induced strain (as depicted in Figs. 2-3), the Pikus-Bir Hamiltonian and the Luttinger-Kohn model [4] together with the computed strain field are employed as usual as an approximation to analyze strain-induced potentials in quantum dots.

The strain-induced potential along the $z$-axis is superimposed to energy band of bulk materials, as shown in Fig. 4. It is found that the strain effects on potentials of both the conduction and valence bands are non-negligible and non-uniform, especially, in the wetting layer and the quantum-dot regions.

Based on the computed energy and the computed wave function spectrum, the energy of interband optical transitions can readily be obtained. The calculated transition energies are in the 


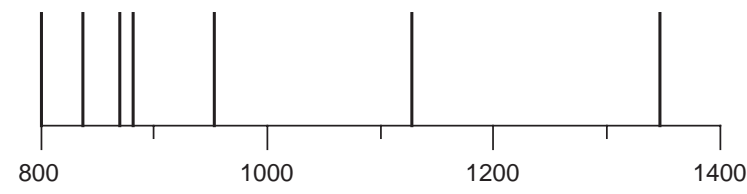

(b)

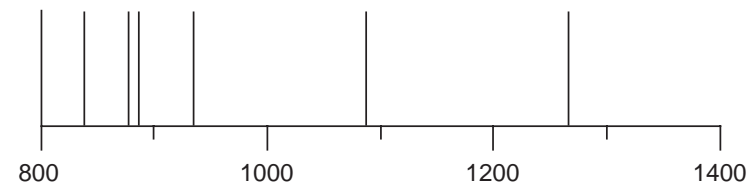

(a)

Fig. 4. The wavelengths of optical transition energies from 836 to $1353 \mathrm{~nm}$ via (a) the conventional model and (b) the proposed model.

range from 1.482 to $0.916 \mathrm{eV}$, which corresponds to $836-1353 \mathrm{~nm}$ in the optical wavelength spectrum as shown in Fig. 4. It shows that the wavelengths based on the strain fields from the proposed model differ significantly from their counterparts through the conventional model, especially for the cases of longer wavelength. It suggests that the proposed model of strain analysis might be necessary for the future optical analysis and applications.

\section{Conclusions}

In this article, a novel model based on the theory of linear elasticity with the aid of finite element analysis has been proposed to investigate the strain field as well as strain effects on optoelectronic properties of the pyramidal InAs/GaAs quantumdot structures. In order to take into account the sequence of fabrication process the strain field in the heterostructure system without capping material was analyzed. The numerical results of the strain field from the proposed model have shown significant difference from the conventional model where the sequence of fabrication process was omitted.

The calculated strain field has also been used as an input for the electronic band structure calculation. The computed wavelengths of the optical transition energies ranged from 836 to $1353 \mathrm{~nm}$. In the meantime, the wavelengths based on the strain fields from the proposed strain analysis have also differed significantly from the counterparts of the conventional strain analysis model. Hence the proposed strain analysis is necessary for the future optical analysis and applications.

\section{Acknowledgement}

This work is carried out in the course of research sponsored by the National Science Council of Taiwan under Grant NSC92-2212-E-002-072.

\section{References}

[1] D. Bimberg, M. Grundmann, N.N. Ledentsov, Quantum Dot Heterostructures, Wiley, New York, 1999.

[2] P. Harrison, Quantum Wells, Wires and Dots: Theoretical and Computational Physics, Wiley, New York, 2000.

[3] G.E. Pikus, G.L. Bir, Sov. Phys. Solid State 1 (1960) 1502.

[4] G. Muralidharan, Jpn. J. Appl. Phys. 39 (2000) L658.

[5] H.T. Johnson, L.B. Freund, C.D. Akyüz, A. Zaslavsky, J. Appl. Phys. 84 (1998) 3714

[6] L.B. Freund, H.T. Johnson, J. Mech. Phys. Solids 38 (2001) 1045. 